\title{
The Drosophila ovary: an active stem cell community
}

\author{
Dániel Kirilly ${ }^{1}$, Ting Xie ${ }^{2}$ \\ ${ }^{1}$ Institute of Genetics, Biological Research Centre of the Hungarian Academy of Sciences, Temesvári krt. 62, Szeged 6726, Hungary; \\ ${ }^{2}$ Stowers Institute for Medical Research, 1000 East 50th Street, Kansas City, MO 64110, USA
}

Only a small number of cells in adult tissues (the stem cells) possess the ability to self-renew at every cell division, while producing differentiating daughter cells to maintain tissue homeostasis for an organism's lifetime. The Drosophila ovary harbors three different types of stem cell populations (germline stem cell (GSC), somatic stem cell (SSC) and escort stem cell (ESC)) located in a simple anatomical structure known as germarium, rendering it one of the best model systems for studying stem cell biology due to reliable stem cell identification and available sophisticated genetic tools for manipulating gene functions. Particularly, the niche for the GSC is among the first and best studied ones, and studies on the GSC and its niche have made many unique contributions to a better understanding of relationships between stem cells and their niche. So far, both the GSC and the SSC have been shown to be regulated by extrinsic factors originating from their niche and intrinsic factors functioning within. Multiple signaling pathways are required for controlling GSC and SSC self-renewal and differentiation, which provide unique opportunities to investigate how multiple signals from the niche are interpreted in the stem cell. Since the Drosophila ovary contains three types of stem cells, it also provides outstanding opportunities to study how multiple stem cells in a given tissue work collaboratively to contribute to tissue function and maintenance. This review highlights recent major advances in studying Drosophila ovarian stem cells and also discusses future directions and challenges.

Cell Research (2007) 17:15-25. doi:10.1038/sj.cr.7310123; published online 2 January 2007

Keywords: Drosophila ovary, germline stem cell, somatic stem cell, escort stem cell

\section{Introduction}

In adult animals, almost every tissue type needs stem cells that are responsible for generating new cells to replace lost cells due to natural cell turnover or injury. Without these stem cells or even with reduced stem cell activities a given tissue would not be able to maintain itself for a long period of time and eventually degenerate due to limited lifespan of differentiated adult cells. Although the proliferative capacity of the stem cells varies widely based on their tissue origin [1], all of them share the ability to regenerate themselves (self-renew) and also to produce functionally differentiated cells for most, if not all, of the organism's lifetime. In contrast with pluripotent embryonic

Correspondence: Ting Xie

Tel: +1-816-926-4061; Fax: +1-816-926-2029;

E-mail: tgx@Stowers-institute.org stem (ES) cells that have the capacity to generate all the cell types in adults, adult stem cells have more restricted lineage potentials. The long-term regenerative potential makes stem cells ideal reagents for treating degenerative diseases such as Parkinson's disease, Alzheimer's disease, diabetes and glaucoma. One of the most exciting discoveries in the field of cancer research is to reveal the existence of cancer stem cells and the potential connection between cancer formation and adult stem cells [2]. Like normal adult stem cells, cancer stem cells can also self-renew but, unlike normal stem cells, they cannot differentiate normally to generate differentiated and functional cells that have the ability to integrate into tissues but instead generate abnormally differentiated cells that drive tumor formation and growth. Therefore, the molecular mechanisms governing normal stem cell self-renewal and differentiation are of great importance for using stem cells in future regenerative medicine, understanding tissue aging and degeneration, and finding new strategies for treatments of cancer. However, 
such mechanisms have just begun to be revealed and remain largely unknown.

One of the major obstacles in stem cell research is to accurately identify stem cells in their native tissue environments due to their rarity and lack of unique molecular markers. In order to clarify the function of a given gene specifically in the stem cell, it is necessary to distinguish stem cells from their progenitors or surrounding cells. Despite the difficulties in identifying stem cells in mammalian tissue, stem cells from several tissues in mice such as skin, blood and intestine have been identified by BrdU labeling [3-5]. Although their regulation has been studied using conditional knockout mice, the process is relatively slow and expensive. In contrast, the Drosophila ovary offers several unique advantages for studying molecular and genetic networks controlling stem cell regulation. First, a small number of easily identified stem cells are located in a simple tubular structure, also known as the germarium, in which stem cells and their surrounding cells are well defined and easily distinguished from one another, facilitating qualitative and quantitative analyses [6-8]. Second, sophisticated but yet elegant genetic and molecular tools make manipulation of gene function in stem cells and their niche easy and precise [7]. Third, more than $50 \%$ of the Drosophila genes have mutations, and microarray and proteomic reagents are available due to the finished Drosophila genome, which dramatically enhance our ability to discover genes that encode intrinsic factors and niche signals important for controlling self-renewal, proliferation and differentiation of stem cells. Finally, comparative studies on the stem cells in the Drosophila ovary and in the testis help find general stem cell regulatory mechanisms because the Drosophila testis also contains easily identified stem cells and niche cells [9]. Therefore, the Drosophila ovary represents one of the best systems for studying basic stem cell biology.

\section{The Drosophila ovary offers unique opportunities for studying three types of stem cells and niches}

The female Drosophila has the remarkable ability to generate a large number of eggs throughout its lifetime because of the existence of permanent stem cells [10]. Each Drosophila female has a pair of ovaries, which is composed of 12 to 16 ovarioles. In each ovariole, the germarium, the most apical structure where stem cells are located, is followed by the developing egg chambers that are arranged in a linear fashion with the most mature egg chamber at the most distal end. In the germarium, there are three types of stem cells, namely germline stem cells (GSCs), somatic stem cells (SSCs) and newly identified escort stem cells (ESCs), whose activities have been confirmed by lineage tracing and laser ablation experiments [11-14] (Figure 1A). The most apical cells in the germarium are a row of 8 to 10 tightly packed disc-like terminal filament (TF) cells, the most posterior one of which directly contacts 5-7 cap cells (Figure 1A). Two groups of stem cells, 2-3 GSCs and 4-6 ESCs, are anchored to cap cells posteriorly, while ESCs also wrap around GSCs with their cellular extensions $[12,14]$. GSCs continuously generate differentiated cystoblasts, which further divide four times synchronously with incomplete cytokinesis to form two-, four-, eight- and 16-cell cysts (Figure 1B). After the cystoblasts move away from the niche, they are wrapped around by differentiated escort cells produced by ESCs. The cysts become fully encased by the escort cells until they reach the stage of 16 cells, and are then surrounded by somatic epithelial follicle cells to bud off the germarium as individual egg chambers. The follicle cells are produced by 2-3 SSCs located in the midway of the germarium [11] (Figure 1C). These stem cells generate several somatic follicle cell lineages, such as polar cells, stalk cells and follicular epithelial cells covering the surface of the growing egg chamber.

Genetic and cell biological studies show that the germarial tip functions as a GSC niche. First, if the two daughters of a GSC following cell division are positioned where GSCs are normally located, both of them adopt the GSC fate [15]. Second, GSCs have to be directly anchored to cap cells through adherens junctions in order to continuously self-renew [16]. Third, TFs and cap cells express several genes that are important for controlling GSC self-renewal [15, 17-22]. One recent study has implicated ESCs as a part of the GSC niche [12]. Thus, the GSC niche is likely composed of TF/cap cells and ESCs, which are all located next to GSCs. In contrast, the SSC niche is potentially composed of TF/cap cells and IGS cells, which is supported by several pieces of experimental evidence. First, TF/cap cells express Hedgehog $(\mathrm{Hh})$ and Wingless $(\mathrm{Wg})$ that are essential for controlling SSC self-renewal [19, 23-25]. Second, Gbb produced by IGS cells also regulates SSC self-renewal [26]. Finally, the anchorage to posterior IGS cells through E-cadherin-mediated cell adhesion is necessary to maintain SSCs [27]. However, it remains unclear what makes the ESC niche. Based on its location and the knowledge of the GSC niche and the SSC niche, we speculate that cap cells and GSCs may form a niche for ESCs since removal of germline cells results in loss of all the IGS cells including ESCs and their progeny [15].

In addition to easily identified stem cells and niches in the Drosophila ovary, the Drosophila system also offers genetic and molecular tools for effectively manipulating functions of genes in both stem cells and niche cells. The FLP-mediated FRT stem cell marking system has been successfully used to generate marked mutant stem cells and 
A
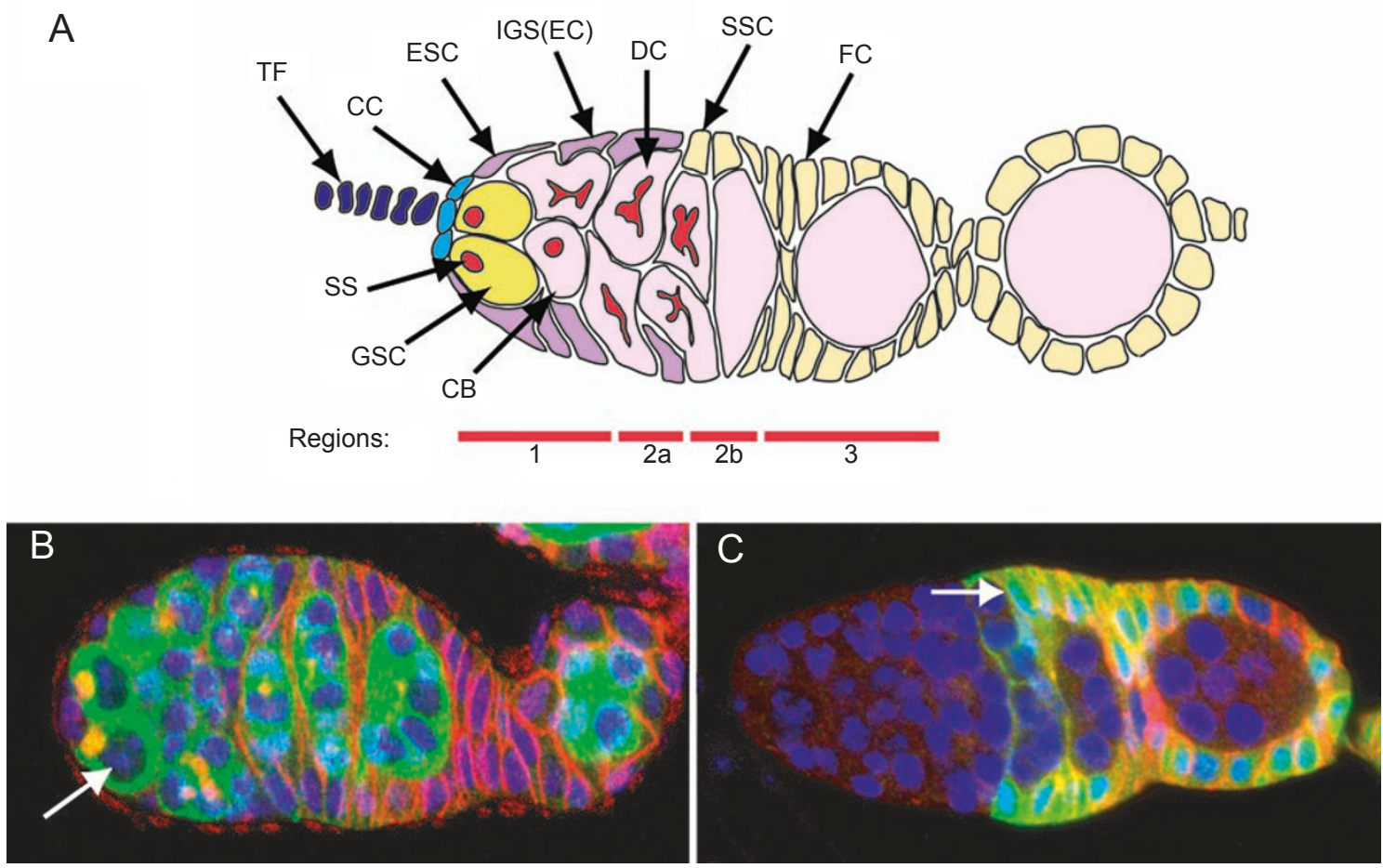

Figure 1 Diagram of the Drosophila germarium and the location of stem cells. (A) TF and cap cells (CC) are the immediate neighbors of the GSC at the anterior end. GSC can be recognized by their intracellular spectrosome (SS). The differentiating daughter cell of the GSC is the cystoblast (CB) that moves posteriorly and becomes encompassed by inner germarial sheath cells (IGS), also called as escort cells. As the CB divides it becomes a 16-cell developing cyst (DC) and it passes through region 2 a, after which it will be surrounded by follicle cells (FC), generated by the SSCs. The three regions of the germarium are indicated below the diagram. (B) GSCs (arrow) are located in the anterior of the germarium. Germline cells are marked by vasa-GFP (green), while cell membranes and the GSC-specific fusome are marked by anti-Hts (red). Note the presence of the spectrosomes in GSCs and their connection to the CCs. (C) Clonally marked SSCs and their differentiating follicle cell progenitors are highlighted by GFP expression (green). SSCs (one indicated by an arrow) are defined as the most anterior labeled cells that are expressing low levels of Fasciclin III (red). Nuclei are stained with DAPI (blue) on $(\mathbf{B}, \mathbf{C})$.

determine the effect of a mutation in a given gene on the maintenance, proliferation and differentiation of GSC, SSC or ESC [12, 17, 27]. This method has been used to demonstrate roles of different pathways and factors in the control of ovarian stem cell regulation in the Drosophila ovary [7]. Recently, MARCM (mosaic analysis with a repressible cell marker) and PMML (positively marked mosaic lineage), two positive marking systems for gene overexpression, have been used to effectively study SSC regulation [26]. In addition, the GAL4-UAS system can be used to overexpress genes, dominant-negative and RNAi constructs to manipulate gene function in a cell-specific manner. For example, nanos-gal4VP16 can drive gene expression specifically in the germline [28], while engrailed-gal4 and c587-gal4 are used to drive gene expression in TF/cap cells and IGS cells, respectively $[18,29]$. GSC-specific genes have been identified using purified GSCs and microarrays [30], while genetic suppressor screens help identify new genes that are important for stem cell regulation [31, 32]. It has recently been shown that Drosophila GSCs can be cultured in vitro, together with somatically derived cells, possibly generated by SSCs [33]. In summary, the Drosophila ovary will continue to provide one of the most effective systems for studying stem cell regulation.

\section{Genes and pathways that control GSC self-renewal, proliferation and differentiation}

Genetic studies in the past decade have identified a number of genes with critical functions in GSC self-renewal, proliferation and differentiation. As expected, some of these genes encode intrinsic factors, including nuclear factors and translational regulators that function inside the GSC, while the others produce proteins that function to generate signals and regulators of signal production within the niche. Several recent reviews have summarized 
the progress in understanding functions of these extrinsic and intrinsic factors in regulation of GSC self-renewal, proliferation and differentiation [6-8, 34]. In this review, we will focus on discussing recent progress in understanding molecular mechanisms governing stem cell regulation in the Drosophila ovary.

Two or three GSCs in the apical tip of the germarium can be reliably identified by their location (direct anchorage to posterior side of the cap cells), size (the biggest cells at the tip of the germarium) and anterior localization of a round spectrosome (Figure 1B). Cap cells can be identified by their unique hh-lacZ and nuclear lamin C. Germ cells including GSC express the Vasa protein, while the spectrosome and its counterpart in the mitotic cyst, the branched fusome, are germ cell-specific organelles that are rich in membrane skeletal proteins such as $\alpha$-Spectrin and $\mathrm{Hu}$ li tai shao (Hts) $[35,36]$. In the Drosophila embryo and the larval brain, neural stem cell-like neuroblasts divide asymmetrically to generate a self-renewing neuroblast and a small-size differentiating ganglion mother cell by specifically segregating differentiation factors into the differentiating daughter cell [37]. Instead of asymmetrically deposited intracellular factors, the asymmetric division of the GSC is accomplished by differential signaling resulting from an asymmetric organization of the GSC niche. Normally, the GSC is anchored to cap cells by adherens junctions formed between the two cell types [16]. As the GSC divides to generate two daughters with the one remaining in contact with cap cells and the other one lying one cell away from the cap cells, the anterior daughter self-renews as a stem cell and the posterior one differentiates into a cystoblast due to differential signaling from their microenvironments. However, the two daughters can adopt the same cell fate, either stem cell or cystoblast, when they are put in the same environment. The two stem cell daughters become GSCs if they both stay in the niche due to available niche space following loss of one neighboring GSC (referred here as symmetric division) [15]; both of them can be turned into cystoblast, resulting in loss of the GSC, if they move away from the niche due to loss of adherens junctions [16]. Such junctions between GSCs and cap cells are established during the niche formation. Interestingly, in the Drosophila testis, adherens junctions also function as focal points for regulating spindle orientation of the stem cell division, assuring the asymmetric cell division [38]. It remains uncertain if adherens junctions participate in controlling asymmetric division of stem cells in other systems as well.

Significant progress has been made in the past few years in gaining a better understanding of how the niche controls GSC function by identifying signaling pathways and genes that regulate these pathways (Figure 2A). The
BMP pathway is the best studied one for controlling GSC function, and is both necessary and sufficient for GSC self-renewal and proliferation $[17,18]$. Two BMP ligands, Dpp and Gbb, are expressed in TF/cap cells, while Gbb is also expressed in IGS cells and follicle cells [18]. The BMP signal produced by the somatic cells directly act on GSCs to control their self-renewal and division [17]. It functions as a short-range signal, which is evidenced by the activation of a BMP response gene, Daughters against $d p p(D a d)$, primarily in the GSC [18, 39-41], and controls GSC self-renewal by directly repressing the expression of differentiation-promoting genes such as bag of marbles (bam) $[18,40]$. BMP signaling also has important roles in controlling the division of primordial germ cells (PGCs) in the developing female gonad and the recruitment of GSCs to their niche [42]. In addition to its role in preventing GSC from differentiation, BMP signaling appears to be capable of reverting differentiated mitotic germ cell clusters into GSCs [43].

Several major questions surrounding the role of the BMP pathway in GSC regulation remain unaddressed: how the short-range effect is controlled, how bam expression is repressed and whether or not there are other repressed target genes. In the Drosophila testis, the BMP signal produced by niche cells also functions as a short-range signal to control GSC self-renewal by repressing bam expression $[44,45]$. Intriguingly, BMP promotes self-renewal of mouse ES cells, but induces differentiation of skin and intestinal stem cells in mice [46-50]. Further studies on GSCs in the Drosophila may help provide insight into how BMP signaling has different functions in different stem cell systems.

Two other potential pathways have also been identified by studying two genes, $f_{s}(1) Y b(Y b)$ and piwi, that are required in somatic cells such as TFs and cap cells. $Y b$ encodes a novel protein [20], while piwi encodes a highly conserved protein involved in regulation of microRNA (miRNA) production [21, 22, 51-54]. Yb exerts its effect on GSC function by regulating expression of piwi and $h h$ in TFs and cap cells [19]. While the piwi-mediated pathway is absolutely essential for GSC self-renewal, Hh signaling plays a non-essential role in controlling GSC self-renewal by having redundant function with the piwi-mediated pathway [19]. Two recent studies have shed some light on the piwi-mediated pathway $[29,55]$. When piwi function is specifically removed from the niche, bam expression is also derepressed in the GSCs like in $d p p$ and $g b b$ mutant GSCs, indicating that piwi also controls bam repression in GSCs. Since piwi functions in the niche cells, it could regulate BMP production, secretion or activation, or it could control the production of a new signal that is also essential for controlling GSC self-renewal and bam repression independently of the BMP pathway. In the future, it 

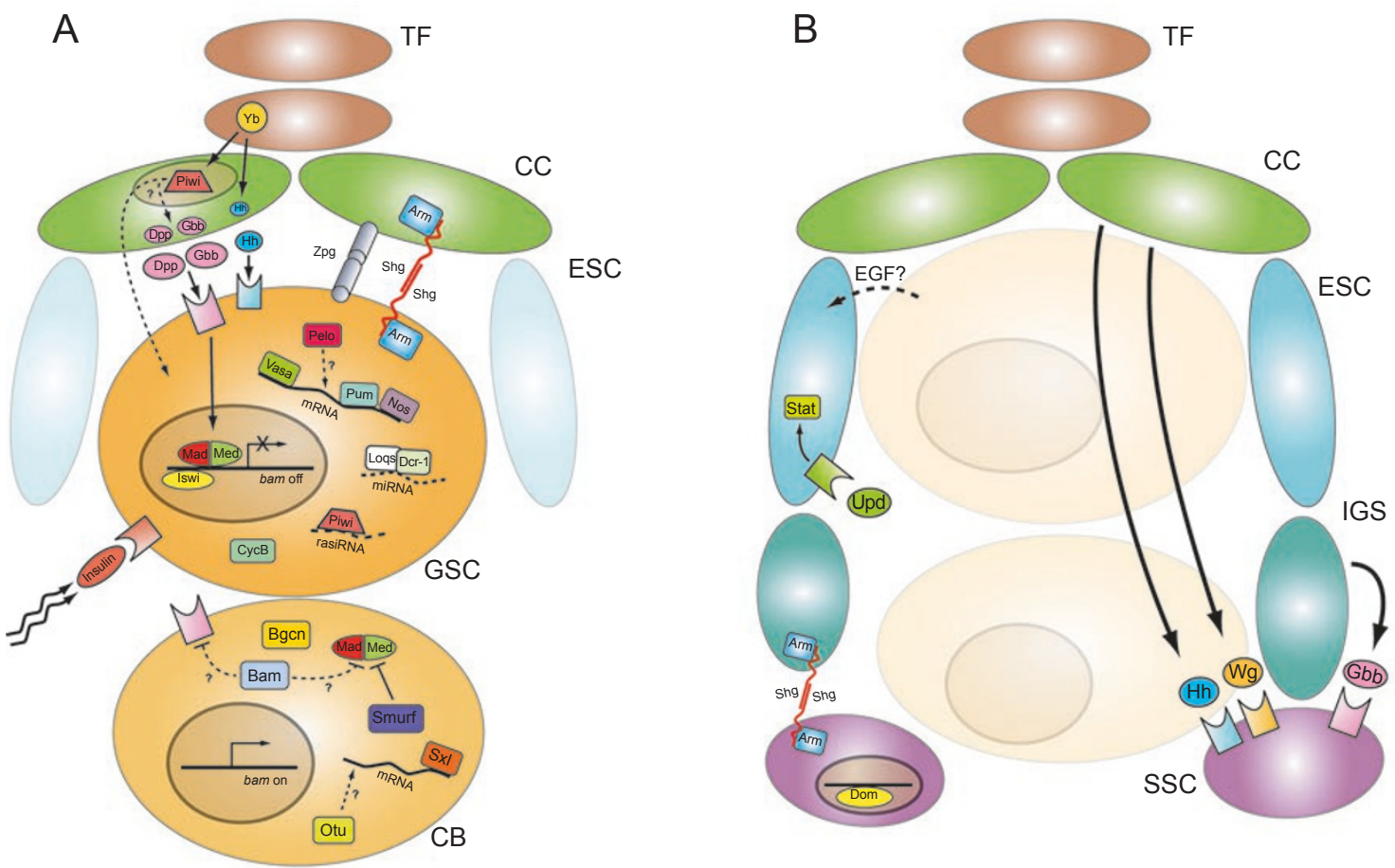

Figure 2 Diagrams of the identified signaling pathways and intrinsic factors that regulate GSC (A), SSC and ESC (B) maintenance and differentiation in the Drosophila ovary. (A) TF/cap cells (CCs) express Yb, Piwi and BMPs (Dpp and Gbb), which control GSC self-renewal and/or division in a non-cell-autonomous fashion, while the insulin pathway controls GSC division. Also, gap junctions formed by Zpg and adherens junctions formed by E-cadherin encoded by shg controls GSC maintenance and anchorage, respectively. The translational regulators (Pum, Nos and Pelo), the cell cycle regulator $\mathrm{CycB}$, the chromatin remodeling factor Iswi are required intrinsically in the GSC to control its self-renewal, while the GSC-expressed Piwi and miRNA pathway components (Dcr-1 and Loqs) are required intrinsically for controlling GSC division. In the cystoblast (CB), Bgcn, Bam, Sxl and Otu control $\mathrm{CB}$ differentiation. (B) Wg and Hh produced by TF/cap cells and Gbb produced by inner germarial sheath (IGS) cells control SSC maintenance, while the Jak-Stat pathway controls ESC maintenance. E-cadherin-mediated cell adhesion is required for anchoring SSCs, while the chromatin remodeling factor, Dom, is required for controlling SSC maintenance.

is important to define the piwi-mediated pathway in the GSC niche in order to gain a better understanding of how the niche controls GSC self-renewal, proliferation and differentiation.

As expected, stem cell activities should also be regulated by the nutritional or health status of the whole organism. In mice, an unknown factor or factors from the young systemic environment can rejuvenate aged muscles and hepatic stem/precursor cells through upregulating the Notch signaling pathway [56]. Two recent exciting studies in the Drosophila ovary have identified insulin as a systemic factor for controlling GSC proliferation [57, 58]. The GSCs defective in insulin signaling divide much slower than wild-type ones. Interestingly, Insulin is not produced by the cells in the germarium but by fat cells in the brain. Insulin is a well-known signal for regulating sugar metabolism and is modulated by nutritional conditions. However, it is not desirable that nutritional conditions affect stem cell self-renewing ability and thus stem cell number. Indeed, the insulin signaling pathway only affects stem cell division but not self-renewal in the Drosophila ovary. It will be interesting to see if insulin signaling also regulates stem cell division in mammalian systems.

Two classes of intrinsic factors have been identified for their opposite effects on GSC self-renewal and differentiation: self-renewal-promoting and differentiation-promoting factors. In addition to BMP downstream components such as receptors and Smads that are required for transducing BMP signal for maintaining self-renewal, several families of proteins have been identified to be required to maintain GSCs. Pumillio (Pum) and Nanos (Nos), two translational repressors, are required for maintaining GSCs in the adult ovary as well as for preventing precocious differentiation of PGCs in the developing female gonad likely by repressing the translation of mRNAs encoding differentiation factors [59-62]. Mutations in both pum and nos cause precocious differentiation of PGCs as well as GSC loss in adulthood $[59,61]$. In addition, a germ cell-specific translation ini- 
tiation factor encoded by vasa (vas) is required for the growth and survival of GSCs [63]. One recent study has also identified a translation release factor-like protein Pelota (Pelo) for its essential role in controlling GSC self-renewal and proliferation [32]. A mutation in pelo causes complete GSC loss within the first week after eclosion and also slows down GSC division. Therefore, translation regulation plays important roles in maintaining GSC self-renewal. In the future, the identification of target mRNAs for Pum, Nos, Vasa and Pelo will further help understand how GSC selfrenewal is controlled.

Three studies have implicated the miRNA pathway in controlling GSC self-renewal and division. The processing and maturation of 19-21 nucleotide miRNAs depends on two dsRNA-binding proteins, Dicer-1 (Dcr-1) and Loquacious (Loqs, also known as R3D1), which are associated with each other [64, 65], while Piwi is known to be involved in generating 29-31 nucleotide repeat-associated small interfering RNAs (rasiRNAs) for silencing retrotransposons and repeated sequences [66]. The piwi mutant GSCs divide much slower than wild-type but have normal self-renewal capacity, indicating that the rasiRNA pathway is required to control GSC division [21]. The marked mutant GSCs for $d c r-1$ have no obvious defects in self-renewal capacity but exhibit dramatic reduction in their division rate due to the delay in the G1-to-S transition [67]. In those mutant GSCs, decapo, encoding a p21-like CDK inhibitor, is upregulated due to the reduced repression of its expression caused by defective miRNA production. Intriguingly, a hypomorphic mutation in loqs causes premature GSC loss and female sterility $[64,65,68]$. It remains unclear if loqs functions inside the GSC to control its self-renewal. If so, it is puzzling that two interacting protein partners that are essential for miRNA processing have distinct functions in controlling GSC function. One of the possible explanations is that $d c r-1$ mutations used in the study are not null alleles and retain enough function for controlling GSC self-renewal but not enough for GSC division. Alternatively, loqs functions in the niche for controlling self-renewal and in the GSC for its division similar to piwi. It is important to distinguish between these two different possibilities and identify responsible miRNAs produced by the niche and GSCs.

Junctional proteins are also important for GSC anchorage in the niche and communication between the niche and GSC. E-cadherin accumulates at the anterior side of the GSC, forming adherens junction with cap cells and maintaining GSC in the niche [16]. Such anchorage is essential for long-term GSC self-renewal since mutations in armadillo and shotgun, encoding $\alpha$-catenin and Ecadherin, the two key components of adherens junctions, respectively, cause rapid GSC loss. Interestingly, cadherin also accumulates between blood stem cells and their niche, suggesting that cadherin-mediated cell adhesion may be a general mechanism for anchoring stem cells in their niche [3]. Gap junctions are also formed between GSCs and cap cells as well as between differentiated germ cells and IGS cells. The mutant GSCs for zero population growth (zpg), encoding the gap junction protein innexin- 4 , are lost, and their progeny cannot differentiate normally, probably due to their inability to transport necessary signals from their surrounding somatic cells.

Two recent studies have identified a cell cycle regulator, Cyclin B ( $\mathrm{CycB})$, and a chromatin remodeling factor, Imitation switch (ISWI), as essential regulators for GSC self-renewal and proliferation $[69,70]$. Although $\mathrm{CycB}$ is generally expressed in the $\mathrm{G} 2$ phase of all dividing cells, $c y c B$ mutant PGCs divide slowly in the developing female gonad, and mutant GSCs fail to be maintained in the adult ovary [69]. It remains unclear at present time how this general cell cycle regulator specifically affects PGC/GSC self-renewal and proliferation. ATP-dependent chromatin remodeling factors, such as ISWI, change the conformation of chromatin, making the DNA accessible to transcription factors for gene activation or repression [71, 72]. The mutant GSCs for iswi are defective in responding to the BMP signal and repressing bam expression and are lost rapidly [70]. Recently, chromatin remodeling factors have been shown to regulate the expression of differentiation promoting genes in human and mouse ES cells $[73,74]$. Future studies on Drosophila ovarian GSCs will provide more insight into how chromatin remodeling factors control stem cell self-renewal in general.

Several differentiation-promoting factors have been identified for their essential roles in promoting cystoblast differentiation as mutations in the genes encoding these factors cause accumulation of GSC-like or cystoblast-like single germ cells (Figure 2A). Among them, bam and benign germ cell neoplasm (bgcn) are the best studied [7578]. bam and bgcn encode a novel protein and a putative RNA-binding protein, respectively, but their biochemical functions remain elusive. Forced expression of bam but not bgcn in GSCs causes their premature differentiation, whereas bam and bgcn mutations cause similar GSC/cystoblast-like tumor phenotypes and genetically interact with each other. One of the bam functions in differentiated cystoblasts is to downregulate BMP signaling through regulating a redundant pathway with the ubiquitin ligase Smurf, which targets phosphorylated Mad (a BMP-specific SMAD) for degradation [41]. In addition, mutations in sex lethal ( $s x l$ ) and ovarian tumor (otu) also cause accumulation of spectrosome-containing single germ cells (either GSC-like or cystoblast-like) and early developing cysts, suggesting that they regulate, but are not absolutely required for, cystoblast differentiation and early cyst division. Sxl is a RNA-bind- 
ing protein, which is known to be involved in regulating mRNA splicing and translational regulation, while Otu is also a putative RNA-binding protein, but its function at the level of mRNA stability, splicing or translation is not clear. Also, it remains unclear if they are sufficient to cause GSC differentiation like bam when they are forced to be expressed in GSCs.

As self-renewal and differentiation are two opposite traits of the stem cell and are precisely balanced in a normal tissue, it would be expected that the factors controlling stem cell self-renewal and the factors controlling stem cell differentiation must counteract with each other to achieve the balance. Indeed, BMP signaling and Piwi-mediated signaling from the niche are required for repressing bam expression in GSCs since defects in BMP signaling and in Piwi-mediated signaling cause bam upregulation in GSCs. Removal of bam function can completely suppress GSC loss phenotype caused by defective BMP or Piwi pathway. Furthermore, the GSC loss phenotype caused by forced bam expression can be partially suppressed by elevated BMP expression [41, 43]. However, in pum and pelo mutant GSCs, bam expression is not upregulated, indicating that Pum and Pelo normally repress the (same or different) Bam-independent pathways [29, 32, 55]. Consistently, bam:pum and bam:pelo double mutant germ cells can differentiate into germ cell cysts, while bam single mutant germ cells fail to differentiate beyond the developmental stage of cystoblast. In order to gain more insight into how self-renewing factors and differentiation-promoting factors interact with each other to control the balance between selfrenewal and differentiation, it is important to learn more about biochemical functions of these factors in GSCs.

\section{Genes and signaling pathways controlling SSC main- tenance and proliferation}

SSCs and GSCs are the two stem cell types that work in a coordinated fashion to efficiently produce egg chambers. As expected, these two stem cell types share some molecular mechanisms controlling self-renewal and proliferation. First, like the GSC, the SSC divides to generate a self-renewing SSC that remains in the niche and a differentiating daughter moving away from the niche for further proliferation and differentiation. Second, like the GSC, the SSC is also anchored to niche cells, the posterior IGS cells, through E-cadherin-mediated cell adhesion [27] (Figure 2B). The SSCs lacking E-cadherin are quickly lost from their niche due to the loss of the contact with the posterior IGS cells. Third, like the GSC, the SSC also requires BMP signaling for its self-renewal and proliferation [26]. The adjacent IGS cells express Gbb/BMP5-8, and major BMP signal transducers are required in SSCs to control their self-renewal and proliferation. Hyperactive signaling prolongs the lifespan of the SSC. Fourth, like the GSC, the SSC also requires the insulin pathway for controlling SSC proliferation [79]. Finally, like the GSC that requires ISWI for its self-renewal, the SSC requires Domino (Dom), an ATP-dependent chromatin remodeling factor, for its selfrenewal [70]. Dom and ISWI belong to the same SWI/SNF family of ATP-dependent chromatin remodeling factors [80]. The mutant SSCs for dom are lost rapidly due to precocious differentiation but not apoptosis [70].

Despite these similarities in mechanisms shared by GSCs and SSCs, there are significant differences between these two cell types. First, the organization of the SSC niche is quite different from that of the GSC niche. The GSC niche is composed of their neighboring cells such as TF/cap cells and ESCs, while the SSC niche includes not only neighboring posterior IGS cells but also TFs/cap cells that are located over a few cells away. Second, $\mathrm{Hh}$ signaling is essential for self-renewal and proliferation of the SSC, while it plays a non-essential role in maintaining the GSC. Hh, which is produced by TFs/cap cells, functions as a long-range morphogen to control SSC self-renewal and proliferation since the SSCs defective in Hh signaling are lost rapidly and hyperactive $\mathrm{Hh}$ signaling increases SSC number [23, 24]. Third, Wg signaling is essential for SSCs, but is dispensable for GSC self-renewal [25]. $\mathrm{Wg}$ is also produced by TFs/cap cells and functions as a long-range signal to directly act on the SSC to control its self-renewal.

Interestingly, in the germarium where all germline cells are removed, SSCs move adjacent to TFs/cap cells following the loss of IGS cells, and continue to proliferate due to the presence of $\mathrm{Hh}, \mathrm{Wg}$ and BMP in TFs/cap cells [11, $15,39]$. Although $\mathrm{Hh}, \mathrm{Wg}$ and BMP pathways are required in the SSC for controlling its self-renewal, little is known regarding how these pathways are integrated in the SSC to regulate expression of target genes that are important for SSC self-renewal and proliferation. Although dom is expressed ubiquitously in the germarium, it is only required in the SSC for its self-renewal [70], and it remains unclear whether it also helps regulating the expression of target genes of the three signaling pathways.

\section{Genes and pathways controlling the maintenance of ESCs and IGS cells}

GSCs and SSCs have been known to be responsible for producing differentiated germ cells and follicle cells, but it remained unknown how IGS cells are maintained until very recently [12]. Decotto and Spradling (2005) showed that the most anterior IGS cells, which directly contact cap cells and also surround GSCs with their cellular processes, 
are also capable of dividing and generating differentiated IGS cells. This group of IGS cells, ranging from four to six cells, is now known as ESCs, and their progeny are renamed as escort cells. It has been proposed that these ESCs divide synchronously with GSCs to generate differentiated escort cells that tightly wrap early germline cells and move posteriorly with the germ cells. This cellular behavior is analogous to cyst progenitor cells in the Drosophila testis, where these cells encompass the developing spermatogonia throughout development [81]. Once germline cysts pass through region 2a of the germarium, they lose their connections to escort cells and become surrounded by the follicle cells [12]. The ESC mutation for dstat, a transcriptional activator downstream of the JAK-STAT pathway, causes the degeneration of the germarium probably due to loss of escort cells, while overexpression of upd, the JAK-STAT ligand, dramatically expands the escort cell population, indicating that JAK-STAT signaling is essential for ESC maintenance and proliferation [12] (Figure 2B). In the germaria in which escort cells are mutant for $d s t a t$, GSCs are also lost rapidly, indicating that ESCs are the part of the GSC niche. In the germarium, there are 40-50 IGS cells [15] that include the estimated 18 escort cells [12]. It remains to be determined if another group of IGS stem cells are designated to generate rest of the IGS cells in the germarium.

In addition to escort cells that wrap around germ cell cysts, the rest of the IGS cells also extend their cellular processes to intimately interact with their underlying germline cysts [82]. Such interactions appear to be essential for normal differentiation of germline cysts since the IGS cells lacking cellular extensions due to a mutation in stem cell tumor (stet), which encodes a rhomboid-like membrane protease that activates EGF ligands in the signaling cell, fail to support differentiation of germline cysts [82]. In stet mutant germaria, undifferentiated germ cells accumulate. In the future, it is important to demonstrate if EGFR signaling is required for producing or maintaining the cellular processes of the escort cells and IGS cells, and identify the signal from the escort/IGS cells that control germ cell differentiation.

\section{Conclusions}

The discovery of the GSC and SSC niches, the identification of GSC and SSC niche signals, and the revelation of the mechanisms of how these signals maintain stem cells in the Drosophila ovary help to provide guidance for studying mammalian stem cells. Studies on three stem cells in the Drosophila ovary have produced several general principles for stem cell biology:

1) The stem cell niche exhibits structural asymmetry, which ensures that one of the two daughters stays in the niche and self-renews, while the other daughter leaves the niche and differentiates. This niche property appears to be universal since all the defined niches in other systems share the same asymmetric feature [83]. Different stem cell types in the same tissue could share the same niche component. For example, GSCs and SSCs share TFs/cap cells as their common niche components.

2) The stem cell is anchored to its niche through cadherin-mediated cell adhesion to ensure long-term self-renewal. Both GSCs and SSCs require E-cadherin-mediated cell adhesion for keeping them in their niches [16, 27]. $\mathrm{N}$-cadherin has been shown to accumulate between blood stem cells and their niche [3].

3) Multiple signals from the niche are required for controlling stem cell self-renewal, and different stem cell types require different combination of niche signals. BMP, $\mathrm{Hh}$ and Piwi-mediated signaling pathways control GSC self-renewal and proliferation, while BMP, Hh and Wg pathways are required for SSC self-renewal and proliferation [7].

4) Different classes of intrinsic factors, translational regulators, chromatin remodeling factors, cell cycle regulators and miRNAs, are required for controlling GSC or SSC self-renewal.

5) Self-renewing intrinsic factors and signaling cascades mediated by niche signals control stem cell self-renewal by repressing the expression of differentiation-promoting genes. For example, BMP- and Piwi-mediated signaling and the chromatin remodeling factor ISWI repress the expression of a differentiation-promoting gene bam in GSCs and thus keep them in an undifferentiated state.

6) The niche can also help to reverse differentiated cells back into stem cells. BMP signaling can reverse differentiated germ cell cysts back into functional GSCs [43]. These generalizations derived from the study of Drosophila ovarian stem cells await further verification from other stem cell systems, particularly mammalian stem cells.

Although much progress has been made in studying Drosophila ovarian stem cells, such as defining stem cell niches, identifying intrinsic factors and niche signals in the control of stem cell self-renewal and differentiationpromoting factors, many important questions still remain to be answered. These questions include: how are different niches built in the Drosophila ovary? How do shared niche components contribute to functions of different niches and coordination of these stem cell activities? What are additional intrinsic factors for stem cell self-renewal and differentiation and extrinsic factors from the niche, particularly for SSCs and ESCs? How do multiple pathways interact with one another to control self-renewal? How do intrinsic factors interact with extrinsic signals to control stem cell self-renewal, proliferation and differentiation? 
These questions can be effectively addressed using a combination of genetic, molecular, biochemical, genomic and cell biological approaches available in Drosophila. The answers to these questions will provide more insights into how Drosophila ovarian stem cells are regulated and how stem cells are controlled in general.

\section{References}

1 Shostak S. (Re)defining stem cells. Bioessays 2006; 28:301308.

2 Clarke MF, Fuller M. Stem cells and cancer: two faces of eve. Cell 2006; 124:1111-1115.

3 Zhang J, Niu C, Ye L, et al. Identification of the haematopoietic stem cell niche and control of the niche size. Nature 2003; 425:836-841.

4 Rochat A, Kobayashi K, Barrandon Y. Location of stem cells of human hair follicles by clonal analysis. Cell 1994; 76:10631073.

5 Bjerknes M, Cheng H. Clonal analysis of mouse intestinal epithelial progenitors. Gastroenterology 1999; 116:7-14.

6 Xie T, Spradling A. The Drosophila ovary: an in vivo stem cell system. In: Marshak DR, Gardner RL, Gottlieb D, eds. Stem cell biology. Cold Spring Harbor, NY: Cold Spring Harbor Laboratory Press, 2001: 129-148.

7 Xie T, Kawase E, Kirilly D, Wong MD. Intimate relationships with their neighbors: tales of stem cells in Drosophila reproductive systems. Dev Dyn 2005; 232:775-790.

8 Lin H. The stem-cell niche theory: lessons from flies. Nat Rev Genet 2002; 3:931-940.

9 Kiger AA, Fuller MT. Male germ-line stem cells. In: Marshak DR, Gardner RL, Gottlieb D, eds. Stem cell biology. Cold Spring Harbor, NY: Cold Spring Harbor Laboratory Press, 2001: 149188.

10 Spradling AC. Developmental genetics of oogenesis. In: Bate M, Martinez Arias A, eds. The development of Drosophila melanogaster. 1st Edition. Cold Spring Harbor: Cold Spring Harbor Laboratory Press, 1993: 1-71.

11 Margolis J, Spradling A. Identification and behavior of epithelial stem cells in the Drosophila ovary. Development 1995; 121:37973807.

12 Decotto E, Spradling AC. The Drosophila ovarian and testis stem cell niches: similar somatic stem cells and signals. Dev Cell 2005; 9:501-510.

13 Wieschaus E, Szabad J. The development and function of the female germ line in Drosophila melanogaster: a cell lineage study. Dev Biol 1979; 68:29-46.

14 Lin H, Spradling AC. Germline stem cell division and egg chamber development in transplanted Drosophila germaria. Dev Biol 1993; 159:140-152.

15 Xie T, Spradling AC. A niche maintaining germ line stem cells in the Drosophila ovary. Science 2000; 290:328-330.

16 Song X, Zhu CH, Doan C, Xie T. Germline stem cells anchored by adherens junctions in the Drosophila ovary niches. Science 2002; 296:1855-1857.

17 Xie T, Spradling AC. Decapentaplegic is essential for the maintenance and division of germline stem cells in the Drosophila ovary. Cell 1998; 94:251-260.
18 Song X, Wong MD, Kawase E, et al. Bmp signals from niche cells directly repress transcription of a differentiation-promoting gene, bag of marbles, in germline stem cells in the Drosophila ovary. Development 2004; 131:1353-1364.

19 King FJ, Szakmary A, Cox DN, Lin H. Yb modulates the divisions of both germline and somatic stem cells through piwi- and hh-mediated mechanisms in the Drosophila ovary. Mol Cell 2001; 7:497-508.

20 King FJ, Lin H. Somatic signaling mediated by fs $(1) \mathrm{Yb}$ is essential for germline stem cell maintenance during Drosophila oogenesis. Development 1999; 126:1833-1844.

21 Cox DN, Chao A, Lin H. piwi encodes a nucleoplasmic factor whose activity modulates the number and division rate of germline stem cells. Development 2000; 127:503-514.

22 Cox DN, Chao A, Baker J, et al. A novel class of evolutionarily conserved genes defined by piwi are essential for stem cell selfrenewal. Genes Dev 1998; 12:3715-3727.

23 Forbes AJ, Lin H, Ingham PW, Spradling AC. Hedgehog is required for the proliferation and specification of ovarian somatic cells prior to egg chamber formation in Drosophila. Development 1996; 122:1125-1135.

24 Zhang Y, Kalderon D. Hedgehog acts as a somatic stem cell factor in the Drosophila ovary. Nature 2001; 410:599-604.

25 Song X, Xie T. Wingless signaling regulates the maintenance of ovarian somatic stem cells in Drosophila. Development 2003; 130:3259-3268.

26 Kirilly D, Spana EP, Perrimon N, Padgett RW, Xie T. BMP signaling is required for controlling somatic stem cell self-renewal in the Drosophila ovary. Dev Cell 2005; 9:651-662.

27 Song X, Xie T. DE-cadherin-mediated cell adhesion is essential for maintaining somatic stem cells in the Drosophila ovary. Proc Natl Acad Sci USA 2002; 99:14813-14818.

28 Van Doren M, Williamson AL, Lehmann R. Regulation of zygotic gene expression in Drosophila primordial germ cells. Curr Biol 1998; 8:243-246.

29 Szakmary A, Cox DN, Wang Z, Lin H. Regulatory relationship among piwi, pumilio, and bag-of-marbles in Drosophila germline stem cell self-renewal and differentiation. Curr Biol 2005; 15:171-178.

30 Kai T, Williams D, Spradling AC. The expression profile of purified Drosophila germline stem cells. Dev Biol 2005; 283:486502.

31 Smulders-Srinivasan TK, Lin H. Screens for piwi suppressors in Drosophila identify dosage-dependent regulators of germline stem cell division. Genetics 2003; 165:1971-1991.

32 Xi R, Doan C, Liu D, Xie T. Pelota controls self-renewal of germline stem cells by repressing a Bam-independent differentiation pathway. Development 2005; 132:5365-5374.

33 Niki Y, Yamaguchi T, Mahowald AP. Establishment of stable cell lines of Drosophila germ-line stem cells. Proc Natl Acad Sci USA 2006; 103:16325-16330.

34 Wong MD, Jin Z, Xie T. Molecular mechanisms of germline stem cell regulation. Annu Rev Genet 2005; 39:173-195.

35 Lin H, Yue L, Spradling AC. The Drosophila fusome, a germline-specific organelle, contains membrane skeletal proteins and functions in cyst formation. Development 1994; 120:947-956.

36 de Cuevas M, Lee JK, Spradling AC. alpha-spectrin is required for germline cell division and differentiation in the Drosophila ovary. Development 1996; 122:3959-3968. 
37 Jan YN, Jan LY. Asymmetric cell division in the Drosophila nervous system. Nat Rev Neurosci 2001; 2:772-779.

38 Yamashita YM, Jones DL, Fuller MT. Orientation of asymmetric stem cell division by the APC tumor suppressor and centrosome. Science 2003; 301:1547-1550.

39 Kai T, Spradling A. An empty Drosophila stem cell niche reactivates the proliferation of ectopic cells. Proc Natl Acad Sci USA 2003; 100:4633-4638.

40 Chen D, McKearin D. Dpp signaling silences bam transcription directly to establish asymmetric divisions of germline stem cells. Curr Biol 2003; 13:1786-1791.

41 Casanueva MO, Ferguson EL. Germline stem cell number in the Drosophila ovary is regulated by redundant mechanisms that control Dpp signaling. Development 2004; 131:1881-1890.

42 Zhu $\mathrm{CH}$, Xie T. Clonal expansion of ovarian germline stem cells during niche formation in Drosophila. Development 2003; 130:2579-2588.

43 Kai T, Spradling A. Differentiating germ cells can revert into functional stem cells in Drosophila melanogaster ovaries. Nature 2004; 428:564-569.

44 Kawase E, Wong MD, Ding BC, Xie T. Gbb/Bmp signaling is essential for maintaining germline stem cells and for repressing bam transcription in the Drosophila testis. Development 2004; 131:1365-1375.

45 Shivdasani AA, Ingham PW. Regulation of stem cell maintenance and transit amplifying cell proliferation by tgf-beta signaling in Drosophila spermatogenesis. Curr Biol 2003; 13:2065-2072.

46 Haramis AP, Begthel H, van den Born M, et al. De novo crypt formation and juvenile polyposis on BMP inhibition in mouse intestine. Science 2004; 303:1684-1686.

47 He XC, Zhang J, Tong WG, et al. BMP signaling inhibits intestinal stem cell self-renewal through suppression of Wnt-betacatenin signaling. Nat Genet 2004; 36:1117-1121.

48 Kobielak K, Pasolli HA, Alonso L, Polak L, Fuchs E. Defining BMP functions in the hair follicle by conditional ablation of BMP receptor IA. J Cell Biol 2003; 163:609-623.

49 Qi X, Li TG, Hao J, et al. BMP4 supports self-renewal of embryonic stem cells by inhibiting mitogen-activated protein kinase pathways. Proc Natl Acad Sci USA 2004; 101:6027-6032.

50 Ying QL, Nichols J, Chambers I, Smith A. BMP induction of Id proteins suppresses differentiation and sustains embryonic stem cell self-renewal in collaboration with STAT3. Cell 2003; 115:281-292.

51 Megosh HB, Cox DN, Campbell C, Lin H. The role of PIWI and the miRNA machinery in Drosophila germline determination. Curr Biol 2006; 16:1884-1894.

52 Grivna ST, Pyhtila B, Lin H. MIWI associates with translational machinery and PIWI-interacting RNAs (piRNAs) in regulating spermatogenesis. Proc Natl Acad Sci USA 2006; 103:1341513420.

53 Kotaja N, Lin H, Parvinen M, Sassone-Corsi P. Interplay of PIWI/ Argonaute protein MIWI and kinesin KIF17b in chromatoid bodies of male germ cells. J Cell Sci 2006; 119:2819-2825.

54 Grivna ST, Beyret E, Wang Z, Lin H. A novel class of small RNAs in mouse spermatogenic cells. Genes Dev 2006; 20:17091714.

55 Chen D, McKearin D. Gene circuitry controlling a stem cell niche. Curr Biol 2005; 15:179-184.

56 Conboy IM, Conboy MJ, Wagers AJ, et al. Rejuvenation of aged progenitor cells by exposure to a young systemic environment.
Nature 2005; 433:760-764.

57 LaFever L, Drummond-Barbosa D. Direct control of germline stem cell division and cyst growth by neural insulin in Drosophila. Science 2005; 309:1071-1073.

58 Drummond-Barbosa D, Spradling AC. Alpha-endosulfine, a potential regulator of insulin secretion, is required for adult tissue growth control in Drosophila. Dev Biol 2004; 266:310-321.

59 Wang Z, Lin H. Nanos maintains germline stem cell self-renewal by preventing differentiation. Science 2004; 303:2016-2019.

60 Forbes A, Lehmann R. Nanos and Pumilio have critical roles in the development and function of Drosophila germline stem cells. Development 1998; 125:679-690.

61 Gilboa L, Lehmann R. Repression of primordial germ cell differentiation parallels germ line stem cell maintenance. Curr Biol 2004; 14:981-986.

$62 \mathrm{Lin} \mathrm{H}$, Spradling AC. A novel group of pumilio mutations affects the asymmetric division of germline stem cells in the Drosophila ovary. Development 1997; 124:2463-2476.

63 Styhler S, Nakamura A, Swan A, Suter B, Lasko P. Vasa is required for GURKEN accumulation in the oocyte, and is involved in oocyte differentiation and germline cyst development. Development 1998; 125:1569-1578.

64 Forstemann K, Tomari Y, Du T, et al. Normal microRNA maturation and germ-line stem cell maintenance requires Loquacious, a double-stranded RNA-binding domain protein. PLoS Biol 2005; 3(7):e236.

65 Jiang F, Ye X, Liu X, et al. Dicer-1 and R3D1-L catalyze microRNA maturation in Drosophila. Genes Dev 2005; 19:16741679.

66 Vagin VV, Sigova A, Li C, et al. A distinct small RNA pathway silences selfish genetic elements in the germline. Science 2006; 313:320-324.

67 Hatfield SD, Shcherbata HR, Fischer KA, et al. Stem cell division is regulated by the microRNA pathway. Nature 2005; 435:974978.

68 Lee YS, Nakahara K, Pham JW, et al. Distinct roles for Drosophila Dicer-1 and Dicer-2 in the siRNA/miRNA silencing pathways. Cell 2004; 117:69-81.

69 Wang Z, Lin H. The division of Drosophila germline stem cells and their precursors requires a specific cyclin. Curr Biol 2005; 15:328-333.

70 Xi R, Xie T. Stem cell self-renewal controlled by chromatin remodeling factors. Science 2005; 310:1487-1489.

71 Corona DF, Langst G, Clapier CR, et al. ISWI is an ATP-dependent nucleosome remodeling factor. Mol Cell 1999; 3:239245.

72 Tsukiyama T, Daniel C, Tamkun J, Wu C. ISWI, a member of the SWI2/SNF2 ATPase family, encodes the $140 \mathrm{kDa}$ subunit of the nucleosome remodeling factor. Cell 1995; 83:1021-1026.

73 Bernstein BE, Mikkelsen TS, Xie X, et al. A bivalent chromatin structure marks key developmental genes in embryonic stem cells. Cell 2006; 125:315-326.

74 Boyer LA, Plath K, Zeitlinger J, et al. Polycomb complexes repress developmental regulators in murine embryonic stem cells. Nature 2006; 441:349-353.

75 Lavoie CA, Ohlstein B, McKearin DM. Localization and function of Bam protein require the benign gonial cell neoplasm gene product. Dev Biol 1999; 212:405-413.

76 Gateff E, Kurzik-Dumke U, Wismar J, et al. Drosophila differentiation genes instrumental in tumor suppression. Int J Dev 
Biol 1996; 40:149-156.

77 McKearin DM, Spradling A. bag-of-marbles: a Drosophila gene required to initiate both male and female gametogenesis. Genes Dev 1990; 4:2242-2251.

78 Ohlstein B, Lavoie CA, Vef O, Gateff E, McKearin DM. The Drosophila cystoblast differentiation factor, benign gonial cell neoplasm, is related to DExH-box proteins and interacts genetically with bag-of-marbles. Genetics 2000; 155:1809-1819.

79 Drummond-Barbosa D, Spradling AC. Stem cells and their progeny respond to nutritional changes during Drosophila oogenesis. Dev Biol 2001; 231:265-278.

80 Ruhf ML, Braun A, Papoulas O, et al. The domino gene of
Drosophila encodes novel members of the SWI2/SNF2 family of DNA-dependent ATPases, which contribute to the silencing of homeotic genes. Development 2001; 128:1429-1441.

81 Fuller MT. Spermatogenesis. In: Bate M, Martinez Arias A, eds. The development of Drosophila. Cold Spring Harbor: Cold Spring Harbor Laboratory Press, 1993: 71-147.

82 Schultz C, Wood CG, Jones DL, Tazuke SI, Fuller MT. Signaling from germ cells mediated by the rhomboid homolog stet organizes encapsulation by somatic support cells. Development 2002; 129:4523-4534.

83 Li L, Xie T. Stem cell niche: structure and function. Annu Rev Cell Dev Biol 2005; 21:605-631. 